Open Access

\title{
Transcranial magnetic stimulation to understand pathophysiology and as potential treatment for neurodegenerative diseases
}

Zhen $\mathrm{Ni}^{1 *}$ and Robert Chen ${ }^{1,2^{*}}$

\begin{abstract}
Common neurodegenerative diseases include Parkinson's disease (PD), Alzheimer's disease (AD), amyotrophic lateral sclerosis (ALS) and Huntington's disease (HD). Transcranial magnetic stimulation (TMS) is a noninvasive and painless method to stimulate the human brain. Single- and paired-pulse TMS paradigms are powerful ways to study the pathophysiological mechanisms of neurodegenerative diseases. Motor evoked potential studied with single-pulse TMS is increased in PD, AD and ALS, but is decreased in HD. Changes in motor cortical excitability in neurodegenerative diseases may be related to functional deficits in cortical circuits or to compensatory mechanisms. Reduction or even absence of short interval intracortical inhibition induced by paired-pulse TMS is common in neurodegenerative diseases, suggesting that there are functional impairments of inhibitory cortical circuits. Decreased short latency afferent inhibition in $A D, P D$ and $H D$ may be related to the cortical cholinergic deficits in these conditions. Cortical plasticity tested by paired associative stimulation or theta burst stimulation is impaired in PD, AD and HD. Repetitive TMS (rTMS) refers to the application of trains of regularly repeating TMS pulses. High-frequency facilitatory rTMS may improve motor symptoms in PD patients whereas low-frequency inhibitory stimulation is a potential treatment for levodopa induced dyskinesia. rTMS delivered both to the left and right dorsolateral prefrontal cortex improves memory in AD patients. Supplementary motor cortical stimulation in low frequency may be useful for HD patients. However, the effects of treatment with multiple sessions of rTMS for neurodegenerative diseases need to be tested in large, sham-controlled studies in the future before they can be adopted for routine clinical practice.
\end{abstract}

Keywords: Alzheimer's disease, Amyotrophic lateral sclerosis, Huntington's disease, Parkinson's disease, Transcranial magnetic stimulation

\section{Background}

Neurodegeneration involves progressive structural and functional loss of specific groups of neurons. The risk of being affected by a neurodegenerative disease increases dramatically with age. With increasing lifespan due to the population-wide health improvements, more individuals will be affected by neurodegenerative diseases in the coming decades. Common neurodegenerative diseases include Parkinson's disease (PD) [1], Alzheimer's disease

\footnotetext{
* Correspondence: nzhen@uhnres.utoronto.ca; robert.chen@uhn.ca

'Division of Neurology, Krembil Neuroscience Centre and Toronto Western

Research Institute, University Health Network, University of Toronto, Toronto, ON, Canada

${ }^{2} 7 \mathrm{MC}-411$, Toronto Western Hospital, 399 Bathurst Street, Toronto, ON M5T
} 258, Canada
(AD) [2], amyotrophic lateral sclerosis (ALS) [3] and Huntington's disease (HD) [4]. The mechanisms underlying neurodegenerative diseases are multifactorial and include genetic and environmental factors. Current treatments for neurodegenerative diseases are symptomatic and there is no accepted disease modifying therapy to slow disease progression [1-4].

Transcranial magnetic stimulation (TMS) is a noninvasive and painless method to stimulate the human brain $[5,6]$. When stimulation is applied to the primary motor cortex (M1), it activates the corticospinal pathway and generates motor evoked potential (MEP) in the target muscles (Fig. 1) [6-8]. In addition to activation of corticospinal neurons, TMS also activates intracortical

\section{Ciomed Central}

(c) $2015 \mathrm{Ni}$ and Chen. Open Access This article is distributed under the terms of the Creative Commons Attribution 4.0 International License (http://creativecommons.org/licenses/by/4.0/), which permits unrestricted use, distribution, and reproduction in any medium, provided you give appropriate credit to the original author(s) and the source, provide a link to the Creative Commons license, and indicate if changes were made. The Creative Commons Public Domain Dedication waiver (http://creativecommons.org/publicdomain/zero/1.0/) applies to the data made available in this article, unless otherwise stated. 


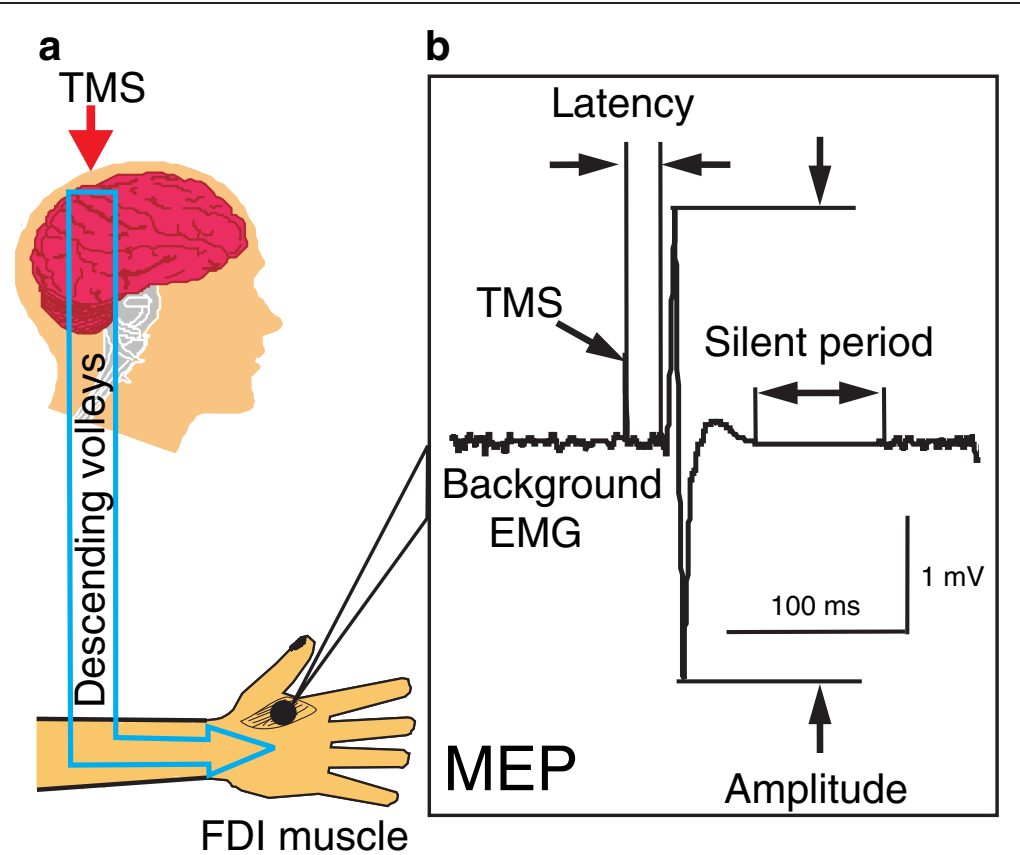

Fig. 1 Transcranial magnetic stimulation and its measurements. a When TMS is applied to the primary motor cortex, it produces descending volleys in the spinal cord. This in turn activates the spinal motoneurons and a motor-evoked potential (MEP) can be recorded in the target muscle (e.g. FDI muscle) with surface EMG. b MEP measurements. When TMS is delivered during voluntary muscle contraction, an MEP is followed by a silent period with no background EMG activity. MEP latency is defined as the time from TMS delivery to the onset of MEP. MEP amplitude is usually measured as the peak-topeak value. Silent period can be measured from the onset or the end of MEP to the first recovery of background EMG activity. EMG = electromyogram, $\mathrm{FDI}=$ first dorsal interosseous, MEP = motor evoked potential, TMS = transcranial magnetic stimulation. Modified from Ni et al., Transcranial magnetic stimulation in different current directions activates separate cortical circuits, Journal of Neurophysiology 2011, 105:749-756 [8]

inhibitory and excitatory neural circuits in the M1. Repetitive TMS (rTMS) refers to application of trains of regularly repeating TMS pulses. These pulses temporally summate to cause changes in neural activity that can outlast the stimulation by minutes to hours [9]. Repeated applications of rTMS can produce even longer effects that last for weeks to months $[7,10]$. Therefore, rTMS may be developed as a therapeutic tool for neurodegenerative diseases [7, 10]. In this article, studies investigating the pathophysiology and focusing on the development of treatments in PD, AD, ALS and HD will be reviewed.

\section{Parkinson's disease}

The motor symptoms of PD largely result from the degeneration of dopaminergic neurons in substantia nigra pars compacta. PD is associated with functional deficits in multiple brain areas, including basal ganglia nuclei, cerebellum and cortical areas [1]. We discuss here these functional deficits as tested by TMS measurements using several experimental designs. The main findings are listed in Table 1.

\section{Single-pulse TMS measurements for Parkinson's disease Motor threshold}

Motor threshold is an important parameter of motor cortical excitability. Rest and active motor thresholds are defined as the minimum TMS intensities that elicit small but reproducible MEPs at rest and during voluntary muscle contraction, respectively [6]. The motor threshold reflects the excitability of the most sensitive group of neurons in the stimulated area in M1. Most studies have reported that rest motor threshold is normal in PD [11-13]. Involuntary contraction caused by tremor and rigidity may affect the measurement in PD. Active motor threshold in PD appears to be normal although a correlation between the degree of bradykinesia and active threshold has been reported [14]. In addition, MEP threshold does not change with medication status [11-13] or deep brain stimulation of the internal globus pallidus [15] or the subthalamic nucleus [16].

\section{MEP amplitude}

MEP amplitude (Fig. 1b) reflects the global excitability of cortical interneurons, corticospinal neurons and spinal motoneurons [7]. Increased MEP amplitude at rest in PD patients has been reported $[17,18]$. Increased MEP amplitude in PD may be related to an imbalance towards disinhibition in the motor pathway. Studies that showed decreased cortical inhibition, increased cortical facilitation and changes in cortical plasticity in PD are discussed below. Patients with internal globus pallidus deep brain 
Table 1 Abnormalities in TMS measurements in neurodegenerative diseases ${ }^{\mathrm{a}}$

\begin{tabular}{|c|c|c|c|c|c|c|}
\hline \multirow[t]{2}{*}{ Measurements } & & \multicolumn{2}{|l|}{ PD } & \multirow[t]{2}{*}{$A D$} & \multirow[t]{2}{*}{ ALS } & \multirow[t]{2}{*}{$\mathrm{HD}$} \\
\hline & & OFF & ON & & & \\
\hline \multirow[t]{3}{*}{ Single-pulse } & MEP threshold & $\circ$ & ० & - & $-1+b$ & + \\
\hline & MEP amplitude & + & + & + & + & - \\
\hline & Silent period & - & ০/+ & - & - & - \\
\hline \multirow[t]{6}{*}{ Paired-pulse } & $\mathrm{SICl}$ & - & $-/ 0$ & - & - & - \\
\hline & ICF & $\circ$ & ० & $\circ$ & + & $\times$ \\
\hline & $\mathrm{LICl}$ & - & - & $\times$ & $\times$ & $\times$ \\
\hline & SAI & $\circ$ & - & - & $\times$ & - \\
\hline & $\mathrm{LAl}$ & - & - & $\times$ & $\times$ & $\times$ \\
\hline & $\| \mathrm{H}$ & $-^{c}$ & $\times$ & - & - & $\times$ \\
\hline \multirow[t]{2}{*}{ Cortical plasticity } & LTP-like effect $^{\mathrm{d}}$ & $-^{e}$ & $\mathrm{o}^{\mathrm{e}}$ & - & $\times$ & - \\
\hline & LTD-like effect $^{d}$ & $\times$ & $\times$ & $-/ 0$ & $\times$ & - \\
\hline
\end{tabular}

Abbreviations: $A D$ Alzheimer's disease, $A L S$ amyotrophic lateral sclerosis, $H D$ Huntington's disease, PD Parkinson's disease, OFF off dopaminergic medication, $O N$ on dopaminergic medication

+ increase; - decrease; $\times$ not tested; $\circ$ normal

ICF intracortical facilitation, IHI interhemispheric inhibition, $L A /$ long latency afferent inhibition, $L I C l$ long interval intracortical inhibition, LTD long-term depression, LTP long-term potentiation, MEP motor evoked potential, SAI short latency afferent inhibition, $\mathrm{SICl}$ short interval intracortical inhibition Notes:

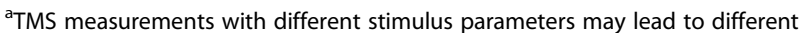
results in testing cortical circuits in neurodegenerative diseases. We only list the most consistent findings in the literatures. Detailed discussion is in the main text of the review

${ }^{\mathrm{b}}$ MEP threshold increased in ALS but could be decreased at early stage of the disease

'Only long latency IHI was decreased in PD patients with mirror movement Such abnormality was found from both the less affected to more affected side and from the more affected to less affected side. Short latency $\mathrm{IHI}$ does not change

${ }^{d}$ LTP-like effects are tested by facilitatory repetitive stimulation protocols including high-frequency repetitive transcranial magnetic stimulation, intermittent theta burst stimulation and paired associative stimulation while LTD-like effects are tested by inhibitory repetitive stimulation protocols including low-frequency repetitive transcranial magnetic stimulation and continuous theta burst stimulation e LTP-like cortical plasticity tested with paired associative stimulation is impaired in PD patients off medication. Dopaminergic medications restore the plasticity in non-dyskinetic patients but not in the dyskinetic patients

stimulation also showed larger MEP amplitude than controls regardless of whether the stimulation was turned on or off [15].

\section{Silent period}

When TMS is applied during voluntary contraction, a disruption of the ongoing muscle activity known as the silent period can be recorded following the MEP (Fig. 1b). The first part of the silent period is partly due to decreased spinal excitability. The latter part of the silent period mainly involves inhibitory effects at the cortical level, mediated by gamma-aminobutyric acid type $B\left(G A B A_{B}\right)$ receptors $[6,19]$. Shortening of the silent period in PD has been reported in many studies [20]. However, such abnormality may not be pronounced at low stimulus intensities [12]. Dopaminergic medication normalizes the shortened silent period in PD [12]. High doses of levodopa may even lengthen the duration beyond the normal range [15].

\section{Tremor reset}

An asymmetric 4-6 Hz resting tremor is a cardinal symptom of PD. Many PD patients also have postural tremor [1]. When stimulation is applied to the motor pathway, the tremor may be transiently disrupted. The reoccurrence of the tremor is then time-locked to the stimulation and this phenomenon is referred to as tremor reset. Mechanical perturbation which modulates spinal reflex pathways has very little effect on postural tremor in PD, suggesting that spinal circuits may not be involved in generating PD postural tremor [21]. TMS applied to M1 completely resets postural tremor in PD [22]. PD rest tremor can also be reset by M1 TMS, suggesting that the M1 is involved in both resting and postural tremor in PD. In addition, cerebellar TMS is effective in resetting the PD postural tremor but not rest tremor, suggesting that the cerebellum is involved in the generation or transmission of postural tremor but not rest tremor in PD [23].

\section{Intracortical circuits in Parkinson's disease}

The excitability of intracortical circuits in M1 can be investigated by a paired-pulse TMS paradigm. The effect of the first conditioning stimulus on the MEP elicited by the second test stimulus depends on the stimulus intensities, the interstimulus interval and the location of conditioning stimulus.

\section{Short and long interval intracortical inhibitions}

Short interval intracortical inhibition (SICI) (Fig. 2) and intracortical facilitation can be tested with both conditioning and test stimuli delivered to the M1, with a subthreshold conditioning stimulus followed by a suprathreshold test stimulus. The test MEP is inhibited at interstimulus interval of 1-5 ms, and facilitated at interval of 7-30 ms [24]. SICI is enhanced by positive allosteric modulators of $\mathrm{GABA}_{\mathrm{A}}$ receptors, suggesting that $\mathrm{SICI}$ is likely mediated by $\mathrm{GABA}_{\mathrm{A}}$ receptors [25-27]. The mechanism mediating intracortical facilitation remains unclear but activation of cortical glutamate circuits may be involved [6]. One early study showed that SICI was reduced in PD patients and levodopa partly normalized this impaired inhibition [12]. Subthalamic nucleus deep brain stimulation increased the reduced SICI both in the on and off medication states [16] while internal globus pallidus stimulation had little effect on SICI [15]. Later studies reported controversial results that SICI was normal in PD patients either on or off medication [28] and decreased SICI was found only at high conditioning intensities [13]. Interestingly, a recent study showed that short interval intracortical facilitation, which is caused by summation of activation of different facilitatory interneurons in the M1, is increased in PD patients 


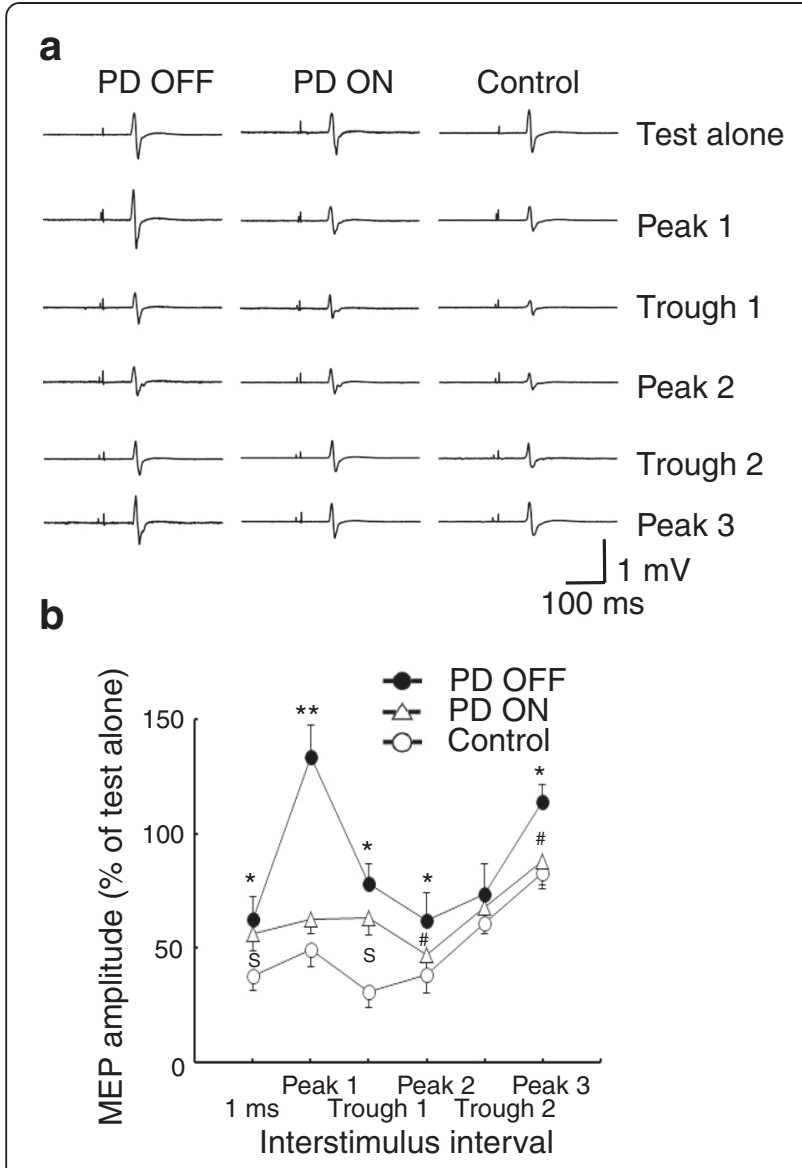

Fig. 2 Abnormal SICI in PD patients. Example of recordings from representative subjects are shown in $\mathbf{a}$. The top row represents the recordings with test stimulus alone and other five rows are recordings for paired-pulse stimulation at different interstimulus intervals. SICl was tested at the interstimulus intervals where short interval intracortical facilitation was at its peaks and troughs. An additional interval of $1 \mathrm{~ms}$ was also tested. Note that SICI was decreased at facilitatory peaks and troughs in the PD OFF medication state, and this was normalized in the PD ON state. The group data analysis is shown in $\mathbf{b}$. The abscissa indicates the interstimulus interval. The ordinate indicates the degree of SICl. It represents the amplitude of paired-pulse induced MEP expressed as a percentage of the MEP amplitude induced by test stimulus alone. Values more than $100 \%$ indicate facilitation and those less than $100 \%$ indicate inhibition. Filled circles indicate MEP in PD patients OFF medication.

Triangles indicate MEP in PD patients ON medication. Open circles indicate MEP in healthy controls. ${ }^{*} p<0.05,{ }^{* *} p<0.01$, comparing PD OFF to control. \# $p<0.05$, comparing PD OFF to PD ON. "S" $p<0.05$, comparing PD ON to control. SICl was reduced in PD OFF compared to controls at an ISI of $1 \mathrm{~ms}$, at short interval intracortical facilitation peak 1 , trough 1, peak 2 and peak 3. Reduced SICI in PD OFF compared to PD ON group was only found at facilitatory peaks. SICI for PD ON was still decreased compared to controls at ISI of $1 \mathrm{~ms}$ and at facilitatory trough 1. $\mathrm{MEP}=$ motor evoked potential, $\mathrm{PD}=$ Parkinson's disease, $\mathrm{SICl}=$ short interval intracortical inhibition. Modified from $\mathrm{Ni}$ et al., Increased motor cortical facilitation and decreased inhibition in Parkinson disease, Neurology 2013, 80:1746-1753 [11]. Promotional and commercial use of the material in print, digital or mobile device format is prohibited without the permission from the publisher Wolters Kluwer Health. Please contact healthpermissions@wolterskluwer.com for further information
[11]. Since the stimulus parameters (interstimulus interval and stimulus intensities) for SICI and short interval intracortical facilitation overlap considerably, decreased SICI (Fig. 2) may partly be explained by increased facilitation in PD [11]. Specifically, short interval intracortical facilitation at the first peak increased from about $200 \%$ of test alone (MEP induced by test stimulus alone) in healthy controls to about $300 \%$ of test alone in PD patients. Concurrently, $\mathrm{SICI}$ at the same interstimulus interval turned from inhibition (about $50 \%$ of test alone) to facilitation (about $130 \%$ of test alone). In addition, SICI was reported to be normal on the less affected side and be reduced on the more affected side in newly diagnosed PD patients [29]. The abnormal SICI with asymmetry was observed up to 1 year after diagnosis [30].

Long interval intracortical inhibition is elicited when a suprathreshold conditioning stimulus is applied 50-200 ms prior to the test stimulus and is likely mediated by $\mathrm{GABA}_{\mathrm{B}}$ receptors [6]. Long interval intracortical inhibition is reported to be decreased in PD [28]. This is consistent with shortened silent period (related to $\mathrm{GABA}_{\mathrm{B}}$ receptors) in PD. Using a triple-pulse TMS paradigm, it has been found that SICI is suppressed in the presence of long interval intracortical inhibition in a manner consistent with reduction in GABA release caused by presynaptic $\mathrm{GABA}_{\mathrm{B}}$ inhibition. The suppressive effect of long interval intracortical inhibition on SICI seen in healthy controls is absent in PD patients. Dopaminergic medications do not normalize this deficit, suggesting that presynpatic inhibition is impaired in $\mathrm{PD}$ and the impairment may be a non-dopaminergic feature of PD [28].

\section{Interhemispheric inhibition}

Interhemispheric inhibition can be measured by two TMS coils placed on bilateral M1s. Both conditioning and test stimuli are suprathreshold. Short and long latency interhemispheric inhibitions peak at interstimulu intervals of $\sim 10$ and $\sim 50$ ms. Inhibition is likely produced by interhemispheric inputs largely mediated through the corpus callosum [31]. There is less long latency interhemispheric inhibition in PD patients with mirror movement than those without mirror movement, suggesting that deficits in transcallosal function may contribute to mirror activity in PD. Such abnormality is found for long latency interhemispheric inhibition from both the less affected to more affected side and from the more affected to less affected side. There is no significant abnormality in short latency interhemispheric inhibition in PD [32].

\section{Afferent inhibition}

Afferent input activated by electrical peripheral nerve stimulation inhibits the contralateral M1. Short (SAI) and long latency afferent inhibition refer to the inhibitory phases at interstimulus intervals of $\sim 20$ and $\sim 200 \mathrm{~ms}$. 
Cholinergic and GABA mediated pathways are involved in generating SAI, whereas transmitter involved in long latency afferent inhibition is not known $[6,8]$. Figure 3 showed that SAI inhibited the MEP induced by test stimulus to about $60 \%$ of its initial size. SAI is normal in PD off dopaminergic medications, but is reduced on medication state (MEP conditioned by electrical peripheral nerve stimulation was about $80 \%$ of test alone). SAI probably represents a direct interaction between the sensory inputs and the M1. This pathway is unaffected by PD but is altered by dopaminergic medication and may contribute to the side effects of dopaminergic drugs. Long latency afferent inhibition is reduced in PD patients independent of their medication status, and probably involves indirect interactions between sensory inputs and the M1 via the basal ganglia or other cortical areas. This defective sensorimotor integration may be a non-dopaminergic manifestation of PD [33]. In addition, reduced SAI in the on

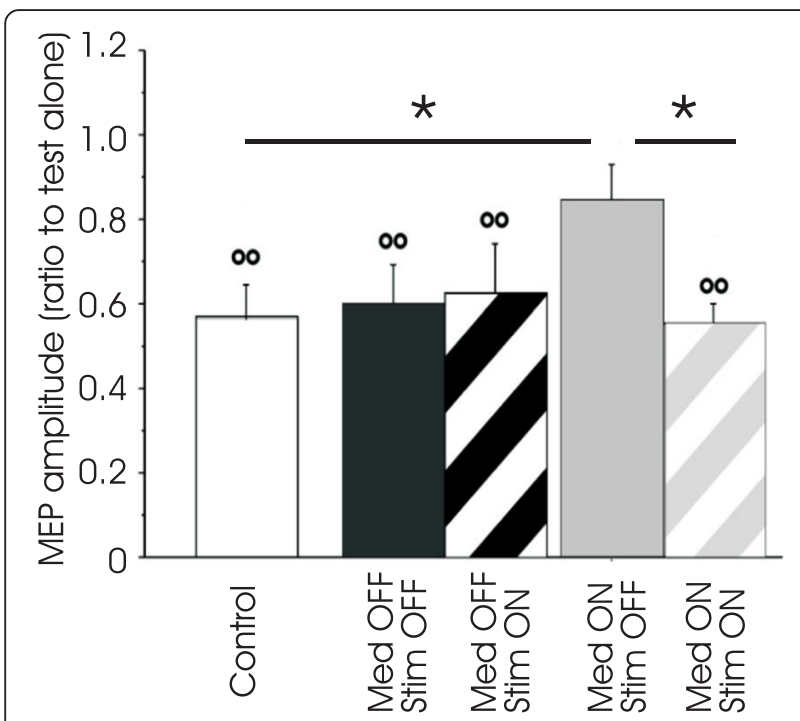

Fig. 3 Short latency afferent inhibition in Parkinson's diseasepatients with subthalamic nucleus deep brain stimulation. The abscissa indicates the different experimental conditions. The ordinate indicates the degree of short latency afferent inhibition. It represents the amplitude of paired-pulse induced MEP expressed as a ratio of the MEP amplitude induced by test alone. Values more than 1 indicate facilitation and those less than 1 indicate inhibition. ${ }^{*} p<0.05$, comparing patients at ON medication OFF stimulation state to healthy controls and patients at ON medication ON stimulation state. The ring asterisks above the columns represent significant inhibition compared to test alone. Note that short latency afferent inhibition was normal in Parkinson's disease patients at OFF medication state while it was reduced at ON medication state. Reduced inhibition at the ON medication state was normalized by the deep brain stimulation. MEP = motor evoked potential. Modified from Sailer et al., Subthalamic nucleus stimulation modulates afferent inhibition in Parkinson disease, Neurology 2007, 68:356-363 [34]. Promotional and commercial use of the material in print, digital or mobile device format is prohibited without the permission from the publisher Wolters Kluwer Health. Please contact healthpermissions@wolterskluwer.com for further information medication state could be restored by subthalamic nucleus deep brain stimulation (Fig.3) and reduced long latency inhibition was partially normalized by the subthalamic stimulation in the on medication state [34]. Furthermore, such normalization of SAI and long latency afferent inhibition with subthalamic nucleus deep brain stimulation only occurred at 6 months but not at 1 month after implantation of stimulation electrodes and these effects were accompanied by normalization of proprioception (spatial and distance errors) [35]. Normalization of afferent inhibition with delayed time course suggests that the effect of subthalamic nucleus deep brain stimulation is related to the plastic changes in basal ganglia and cortical circuits produced by the chronic stimulation. In addition, the modulation of intracortical circuits by afferent inputs can be tested with a triple-pulse TMS paradigm. While long interval intracortical inhibition is reduced by long latency afferent inhibition in healthy controls, such modulation of long interval intracortical inhibition by afferent inputs is impaired in PD patients in both off and on medication states, which is manifested as similar degree of long interval intracortical inhibition in the presence of long latency afferent inhibition compared to that without afferent inhibition [33].

\section{Cerebellar inhibition}

Cerebellar inhibition refers to the phenomenon that stimulation over the cerebellum suppresses the MEP produced by contralateral M1 TMS delivered 5 to $7 \mathrm{~ms}$ later. Cerebellar inhibition is mediated by the cerebellothalamocortical pathway. Cerebellar TMS activates cerebellar Purkinje's cells that inhibit the deep cerebellar nuclei, which has an excitatory projection to the motor cortex via the ventral thalamus [6]. Cerebellar inhibition is decreased in PD. Decreased inhibition correlated with the degree of reset of postural tremor caused by cerebellar stimulation, suggesting that the deficits on the cerebellothalamocortical pathway may be related to the tremor generation in PD [23].

\section{Connectivity between the basal ganglia and M1}

Inputs from the basal ganglia modulate M1 excitability. In PD patients with subthalamic nucleus deep brain stimulation, subthalamic stimulation leads to cortical evoked potential on the scalp with peak latencies of $\sim 3$ and $\sim 20 \mathrm{~ms}$ [36]. Moreover, single pulse subthalamic stimulation produced two phases of MEP facilitation at 2-4 ms and 21-24 ms after the stimulation. The time course of MEP facilitation coincides with that of the evoked potentials recorded at the scalp. Antidromic conduction along the corticosubthalamic pathway likely mediates the early phase of facilitation while the late phase is likely mediated by synaptic transmission through the basal ganglia-thalamo-cortical circuit [36]. 


\section{Cortical plasticity in Parkinson's disease}

Cortical plasticity can be tested by paired associative stimulation, which involves repetitive application of electrical peripheral nerve stimulation followed by TMS to M1. If peripheral stimulation precedes TMS by $\sim 25 \mathrm{~ms}$, the two stimuli arrive at the M1 at about the same time and lead to MEP facilitation in M1 [37]. This type of cortical plasticity is impaired in PD patients off medication. Dopaminergic medications restore the plasticity induced by paired associative stimulation in non-dyskinetic PD patients but not in the dyskinetic PD patients, suggesting that the development of dyskinesia is associated with greater disturbance of cortical plasticity [38]. In more advanced PD patients implanted with subthalamic nucleus deep brain stimulation, restoration of plasticity with paired associative stimulation was only observed in the medication on and stimulation on state (Fig. 4) [39]. Specifically, MEP amplitude 30 and $60 \mathrm{~min}$ after the paired associative stimulation increased to about $150 \%$ of that at baseline in healthy controls. In patients with either medication off or deep brain stimulation off, MEP amplitude after paired associative stimulation was still about $100 \%$ of baseline. When the patients were at both stimulation and deep brain stimulation on state, MEP after paired associative stimulation was facilitated to the similar level to that in healthy controls (about $150 \%$ of baseline). The result suggests that the restoration of cortical plasticity is related to the clinical benefits of deep brain stimulation in PD. On the other hand, MEP facilitation induced by paired associative stimulation on the less affected side in the newly diagnosed PD patients was increased while the same protocol did not produce MEP facilitation on the more affected side in these patients [29]. Furthermore, the asymmetric responses to paired associative stimulation was found up to one year after diagnosis and the degree of asymmetry correlated with asymmetry in clinical rating scores for the less and more affected sides [30]. Intermittent theta burst stimulation produces MEP facilitation in healthy subjects [40]. Similar MEP facilitation has been reported in PD patients [41] whether in the medication on or off state [42]. However, this form of cortical plasticity may be impaired in more advanced PD patients [43].

\section{Therapeutic rTMS in Parkinson's disease}

rTMS involves trains of TMS pulses delivered with durations ranging from several seconds to several minutes at various frequencies and intensities. The effects of these

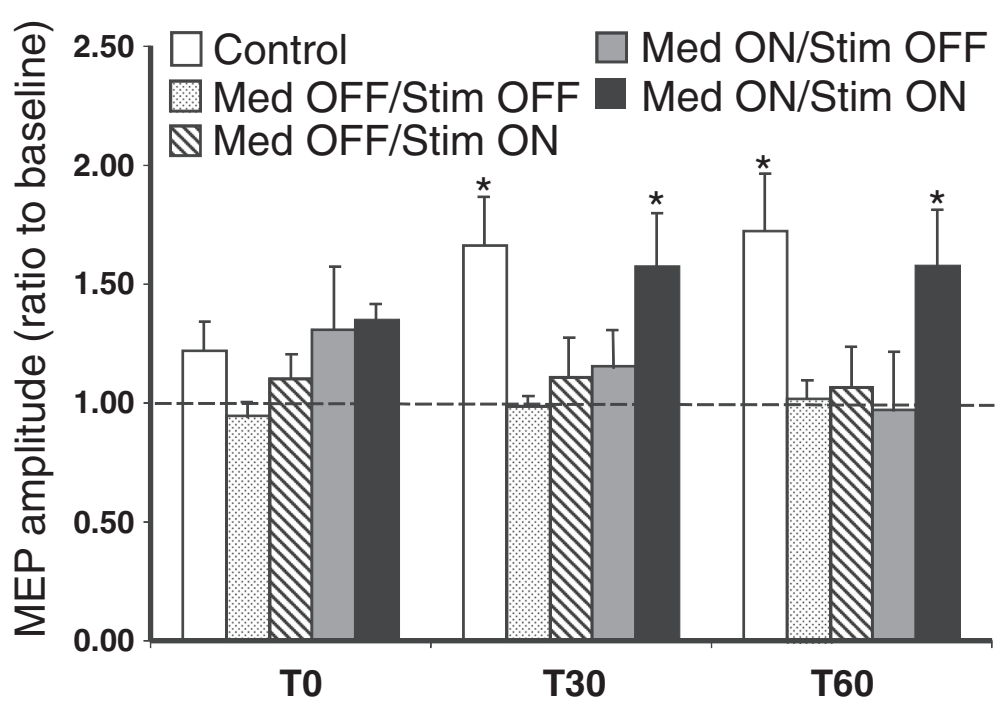

Fig. 4 Motor cortical plasticity induced by paired associative stimulation in Parkinson's disease with subthalamic nucleus deep brain stimulation. The abscissa indicates the time points $(0,30$ and $60 \mathrm{~min}$ ) after the intervention of paired associative stimulation. The ordinate indicates the MEP amplitude after the intervention. The values are expressed as a ratio to the MEP amplitude at baseline (before intervention). Values more than 1 indicate facilitation and those less than 1 indicate inhibition. White columns represent healthy controls. Columns with dots represent patients at medication OFF and deep brain stimulation OFF state. Hatched columns represent patients at medication OFF and stimulation ON state. Grey columns represent patients at medication ON and stimulation OFF state. Black columns represent patients at both medication and stimulation ON state. Note that cortical plasticity was impaired in the patients compared to healthy controls. Impaired cortical plasticity was only restored at the medication ON and deep brain stimulation ON state. ${ }^{*} p<0.05$, comparing MEP at different time points to that at baseline (before intervention). MEP = motor evoked potential. Modified from Kim et al., Effects of subthalamic nucleus stimulation on motor cortex plasticity in Parkinson disease, Neurology 2015, 85:425-32 [39]. Promotional and commercial use of the material in print, digital or mobile device format is prohibited without the permission from the publisher Wolters Kluwer Health. Please contact healthpermissions@wolterskluwer.com for further information 
pulses temporally summate to cause greater and longer duration of changes in neural activity than those from single-pulse TMS. Generally, high-frequency rTMS potentiates MEP and low-frequency rTMS suppresses MEP when delivered to the M1 [6,7]. Since the effects of a single session of rTMS can last for several hours and repeated sessions may last for months, rTMS is a potential treatment for neurological disorders.

While many studies investigated the effects of rTMS on PD symptoms, the results were variable [44]. A large placebo effect with sham stimulation has been observed [45]. Meta-analyses found that high-frequency rTMS improved motor symptoms in PD patients while lowfrequency rTMS had little benefit [44, 46] (Table 2). Intermittent theta burst stimulation has also been used to treat PD motor symptoms. However, a study that used eight sessions of stimulation over two weeks did not find long-term effect on PD motor symptoms but there were benefits on mood [41]. Low-frequency rTMS has been used to treat levodopa induced dyskinesia. One $\mathrm{Hz}$ rTMS over the M1 [47] with a two-week course produced short term improvement in levodopa induced dyskinesia [48]. Similar improvement was confirmed by a sham-controlled study. However, significant improvement in dyskinesia after rTMS was only found when compared to baseline and the difference between real and sham stimulations was not significant [49].

Stimulation of other areas outside the M1 may also be effective. In particular, a sham-controlled study with a relatively large sample size reported that $5 \mathrm{~Hz}$ rTMS applied to the supplementary motor area significantly improved

Table 2 Therapeutic repetitive TMS protocols for neurodegenerative diseases

\begin{tabular}{|c|c|c|c|}
\hline & Protocol $^{a}$ & Target & $\begin{array}{l}\text { Potential beneficial } \\
\text { effects }\end{array}$ \\
\hline \multirow[t]{2}{*}{$\begin{array}{l}\text { Parkinson's } \\
\text { disease }\end{array}$} & Facilitatory & $\begin{array}{l}\text { M1, SMA, } \\
\text { PMd }\end{array}$ & $\begin{array}{l}\text { Improve motor symptoms } \\
\text { mood }^{\mathrm{b}}\end{array}$ \\
\hline & Inhibitory & $\begin{array}{l}\text { M1, } \\
\text { cerebellum }\end{array}$ & $\begin{array}{l}\text { Improve levodopa induced } \\
\text { dyskinesia }\end{array}$ \\
\hline $\begin{array}{l}\text { Alzheimer's } \\
\text { disease }\end{array}$ & Facilitatory & DLPFC & $\begin{array}{l}\text { Improve memory, } \\
\text { cognition }\end{array}$ \\
\hline $\begin{array}{l}\text { Amyotrophic } \\
\text { lateral sclerosis }\end{array}$ & Inhibitory & M1 & Improve motor symptoms \\
\hline $\begin{array}{l}\text { Huntington's } \\
\text { disease }\end{array}$ & Inhibitory & SMA, M1 & Improve chorea \\
\hline
\end{tabular}

Abbreviations: DLPFC dorsolateral prefrontal cortex, $M 1$ primary motor cortex, $P M d$ dorsal premotor cortex, SMA supplementary motor area Notes:

${ }^{a}$ Facilitatory protocols include high-frequency repetitive transcranial magnetic stimulation and intermittent theta burst stimulation; inhibitory protocols include low-frequency repetitive transcranial magnetic stimulation and continuous theta burst stimulation

${ }^{\mathrm{b}}$ Facilitatory protocols with different stimulus parameters applied to M1, SMA and PMd may improve motor symptoms in PD

${ }^{\mathrm{C}} \mathrm{A}$ study of eight sessions of intermittent theta burst stimulation of M1 over two weeks reported benefits in mood in PD the clinic rating scores and bradykinesia in PD patients [50]. Continuous theta burst stimulation, a type of inhibitory rTMS, delivered to the cerebellum improved levodopa induced dyskinesia in PD [51]. In addition, $5 \mathrm{~Hz}$ rTMS over dorsal premotor cortex facilitated MEP in healthy controls but not in PD patients off medications. After levodopa administration, the facilitatory effect of premotor cortical stimulation on the motor cortex was restored [52].

\section{Alzheimer's disease}

$\mathrm{AD}$ is the most common form of dementia and is characterized by progressive neuronal degeneration. The degenerative process leads to atrophy initially in the hippocampus and entorhinal cortex, then progressively expanding into wide areas including the cerebral cortex and subcortical regions $[2,53]$. Mild cognitive impairment $(\mathrm{MCI})$ is considered a transitional stage between normal aging and clinically probable AD. The functional impairments in $\mathrm{AD}$ measured with TMS paradigms are summarized in Table 1.

\section{Single-pulse TMS measurements for Alzheimer's disease Motor threshold}

Rest motor threshold is decreased in AD [54]. However, the threshold is preserved in patients with early disease [55] and in patients with MCI [56], suggesting that reduction in rest threshold may be a compensatory mechanism for the neuronal loss in motor cortical areas and may reflect a functional change in these areas with disease progression. Reduction in active motor threshold in $\mathrm{AD}$ has also been reported [54].

\section{MEP amplitude and silent period}

MEP amplitude may be normal at early stage of AD [54] but is increased in patients at advanced stages [57]. Interestingly, a TMS mapping study showed that the hotspot did not change while the center of gravity for MEP amplitude shifted in a fronto-medial direction in patients with mild to moderate $\mathrm{AD}$, suggesting an early cortical reorganization in AD [58]. Silent period is shortened in moderate to severe $A D$, suggesting that $A D$ may impair the function of $\mathrm{GABA}_{\mathrm{B}}$ receptor mediated inhibitory circuits in M1 at late disease stages [59].

\section{Intracortical circuits in Alzheimer's disease Short latency afferent inhibition}

Reduction in SAI is significant at many disease stages in AD [54, 56, 60-64] and this is consistent with postmortem studies showing central cholinergic impairment in AD [53]. Decreased SAI correlated with the degree of memory loss [60] and the degree of euphoric manic state in $\mathrm{AD}$ [61]. These correlations may be explained by the cholinergic dysfunction in temporo-limbic areas such as hippocampus, entorhinal cortex and amygdala. Administration of 
a single dose of rivastigmine (an acetylcholinesterase inhibitor) restored the decreased SAI in AD [54]. Since decreased SAI was found in early $\mathrm{AD}[62]$ and even in amnesic MCI patients $[63,64]$, it is a potential biomarker for the diagnosis of AD.

\section{Other intracortical circuits}

Reduction in SICI has been reported [65]. The degree of disinhibition correlated with the severity of AD [65]. However, other studies reported no difference in SICI between patients and controls [54, 57]. Although AD may be related to changes in cortical glutamatergic transmission $[53,58]$, intracortical facilitation in $\mathrm{AD}$ and MCI patients were normal [54, 57, 64]. Interhemispheric inhibition is decreased in amnesic MCI patients [64]. However, decreased inhibition does not correlate with the scores of mini-mental status examination or reduced SAI, suggesting that structural or functional impairment in transcallosal connection may occur earlier than the cognitive impairments in MCI [64].

\section{Cortical plasticity in Alzheimer's disease}

Long term potentiation-like cortical plasticity is impaired in AD. Five Hz rTMS which produced MEP increase in healthy controls decreased MEP in $\mathrm{AD}$ patients [66]. Similarly, paired associative stimulation [67] and intermittent theta burst stimulation [68], which induce MEP facilitation in normal subjects, also led to reduced cortical excitability in $\mathrm{AD}$ patients. Whether long term depression-like effect is altered in $\mathrm{AD}$ is controversial. One $\mathrm{Hz}$ rTMS, which produced MEP inhibition in healthy controls [47], had no effect in AD patients [69]. However, MEP inhibition with continuous theta burst stimulation in AD was normal [68].

\section{Therapeutic rTMS for Alzheimer's disease}

The assumption in AD that memory deficit is related to functional impairment in dorsolateral prefrontal cortex [70] makes this cortical area a common target of therapeutic intervention (Table 2). It was reported that application of $20 \mathrm{~Hz}$ rTMS to both the left and right dorsolateral prefrontal cortex improved the accuracy of an action naming task in both mild and moderate to severe $A D$ patients [71]. A subsequent study with daily $20 \mathrm{~Hz}$ rTMS with 2000 pulses applied to the left dorsolateral prefrontal cortex for 2 or 4 weeks showed longlasting improvement (8 weeks) in language comprehension in moderate $\mathrm{AD}$ patients [72]. Another study reported that $20 \mathrm{~Hz}$ right side followed by left side dorsal lateral prefrontal cortical stimulation applied for 5 days improved the score of mini-mental status examination in $\mathrm{AD}$ patients. On the other hand, $1 \mathrm{~Hz}$ stimulation applied in the same order (right followed by left side stimulation) had no effect, suggesting that facilitatory but not inhibitory stimulation has therapeutic effects in AD [73]. However, another study reported that a single session of inhibitory $1 \mathrm{~Hz}$ rTMS over right dorsolateral prefrontal cortex increased the recognition memory performance in both healthy controls and MCI patients [74].

\section{Amyotrophic lateral sclerosis}

ALS is a rapidly progressive neurodegenerative disorder of the motoneurons in the M1, brainstem and spinal cord. A combination of upper and lower motoneuron dysfunction comprises the clinical ALS phenotypes [3].

\section{Single-pulse TMS measurements in amyotrophic lateral sclerosis}

MEP threshold is increased in ALS [75, 76] (Table 1). However, a longitudinal study reported reduced MEP threshold at early stage of the disease, which may explain muscle fasciculation with motor neuronal changes at this stage [76]. Central motor conduction time is prolonged in ALS, reflecting axonal degeneration of the fast conducting fibers of corticospinal neurons [76]. MEP amplitude increases in sporadic [77] and familial forms of ALS [78], prominently in the early stage of the disease. In addition, MEP amplitude correlates with traditional measurement of peripheral nerve functions (compound muscle action potential) and with measurement for axonal excitability in ALS, suggesting an association between cortical hyperexcitability and motoneuron degeneration [77]. Reduction in duration of silent period is also prominent at early stage of ALS, indicating degeneration or dysfunction of inhibitory interneurons with reduced $\mathrm{GABA}_{\mathrm{B}}$ receptor functions in ALS [77, 78].

\section{Intracortical circuits in amyotrophic lateral sclerosis}

SICI is reduced or absent in ALS [77-79] (Table 1). This is consistent with the pathological finding of degeneration of inhibitory cortical interneurons in ALS [80]. In addition, reduction in SICI precedes the clinical development of familial ALS, which may help in establishing the diagnosis [78]. Intracortical facilitation is increased in ALS $[77,78]$, suggesting that glutamate mediated excitotoxicity may be involved in motoneuron hyperexcitability. Involvement of glutamate circuit in ALS pathophysiology is supported by the interesting finding that glutamate antagonist riluzole restored the decreased SICI in ALS patients [79]. Interhemispheric inhibition is also decreased in ALS [81]. Taken together, the reduction in cortical inhibition and increase in cortical facilitation may be related to hyperexcitability of cortical motoneurons in ALS patients.

\section{Cortical plasticity and therapeutic rTMS for amyotrophic lateral sclerosis}

Two weeks of daily sessions of $5 \mathrm{~Hz}$ rTMS only had transit benefit on motor performance and the quality of 
life in ALS patients [82]. Twenty Hz rTMS even showed a tendency to accelerate disease progression [83]. These studies suggest that facilitatory rTMS may have minor beneficial effects or may be harmful in some circumstances in ALS. Inhibitory $1 \mathrm{~Hz}$ rTMS showed slight benefits in two ALS patients [83], supporting the idea that down regulation of hyperexcited motoneurons may improve symptoms (Table 2). Subsequent studies by the same group tested the effect of inhibitory rTMS with a design delivering 5 consecutive daily sessions of continuous theta burst stimulation per month. Long term benefit was observed in studies with different durations (0.5-2 years) and different sample sizes. A 26-month trial in a single case reported a slower rate of deterioration with stimulation compared to baseline. The strongest beneficial effect was found in the first 12 months with stimulation [84]. A six-month study reported a slight but significantly slower disease progression in 7 patients with real stimulation compared to 8 patients with sham stimulation [85]. Unfortunately, a one-year follow up double blinded placebocontrolled study with more patients failed to confirm the positive effects of the previous studies [86].

\section{Huntington's disease}

HD is a genetic neurodegenerative disease due to pathological expansion of the triplet cytosine-adenine-guanine (CAG) repeat in the Huntingtin gene in chromosome 4, which results in an excessively long polyglutamine stretch in protein Huntingtin and eventually causes loss of GABAergic neurons in striatum [4]. HD is characterized by a triad of symptoms with motor, cognitive and psychiatric disturbances.

Single- and paired-pulse TMS measurements in Huntington's disease

Higher rest and active motor thresholds and smaller rest MEP size compared to healthy controls were found in both very early symptomatic HD patients and HD gene carriers [87]. However, probably due to the small sample size and phenotypic heterogeneity, other studies found no difference in MEP threshold [88, 89] or amplitude [88] between HD patients and controls (Table 1). Although silent period may be normal at the early or preclinical stage of HD [87], progressive shortening in silent period with functional decline was found in symptomatic patients at two-year follow up [90]. The finding is consistent with $\mathrm{HD}$ pathology with GABAergic neuronal loss in the brain and suggests that the silent period may be a potential biomarker of the disease progression. Several studies reported normal SICI in symptomatic HD patients $[89,91]$. However, the results may be confounded by inclusion of patients with chorea due to various etiologies. The conditioning stimulus intensity for producing same degree of SICI was found to be increased in early and even in the preclinical stage of the disease [87]. SAI was decreased in the same group of patients [87]. These studies with singleand paired-pulse measurements support the view that cortical functional impairments occur early in HD.

\section{Cortical plasticity and therapeutic rTMS in Huntington's disease}

Cortical plasticity is impaired in HD. MEP facilitation produced both by $5 \mathrm{~Hz}$ rTMS [92] and by paired associative stimulation [93] were reduced in HD patients. MEP inhibition produced by continuous theta burst stimulation was decreased in early symptomatic HD patients and HD gene carriers [88]. The use of rTMS as a treatment for $\mathrm{HD}$ has been studied (Table 2). One $\mathrm{Hz}$ but not $5 \mathrm{~Hz}$ rTMS applied to the supplementary motor area reduced chorea scores in HD patients, suggesting that suppression of supplementary motor cortical excitability may lead to improvement in HD symptoms [94]. Interestingly, dramatic improvement in dyskinesia lasting for $24 \mathrm{~h}$ after a single session of continuous theta burst stimulation to M1 was reported in a case of hemichorea secondary to midbrain and caudate hemorrhage [95].

\section{Conclusions and final remarks}

Although aging is the greatest risk factor for neurodegenerative diseases, many neurodegenerative diseases can be caused by genetic mutations and are associated with protein misfolding and degradation. The effects of neurodegeneration can be found in many different levels of neuronal circuitry ranging from the molecular level to the systems level. Studies using animal models and neuroimaging techniques are searching for the biomarkers for neurodegenerative diseases. Development of disease modifying therapies such as gene therapy, stem cell transplant and neuroprotective agent are actively being pursued [96].

TMS provides a non-invasive and powerful process to investigate the synaptic activity and to manipulate the synaptic plasticity in human cortex at the systems level. Studies with single- and paired-pulse TMS showed abnormal cortical excitability in patients with neurodegenerative diseases. rTMS within established guidelines is safe for the patients with neurodegenerative diseases and showed symptomatic benefit in some studies. Several major issues should be considered for future studies that focus on better understanding of the pathophysiology and novel therapeutics for neurodegenerative diseases. First, the protocols with diagnostic or therapeutic potentials should be translated into clinically practical applications. Currently, this is largely limited by the fact that many TMS measurements have large within-subject and between-subject variations [6,7]. Second, there is no current biomarker which can confirm the diagnosis of neurodegenerative disease at early stage and monitor the 
disease progression. Recently, genetic (such as genome sequencing, proteomics) and neuroimaging (such as positron emission tomography, functional magnetic resonance imaging) approaches are being undertaken to identify potential biomarkers for neurodegenerative diseases. Future studies combining TMS with these techniques may provide new opportunity to find clinically useful biomarkers for neurodegenerative diseases. Third, the current evidence showed that the beneficial effects of rTMS for neurodegenerative diseases are mild to moderate and short-lasting. While multiple sessions of rTMS may extend the clinical benefit, development of rTMS into a practical treatment requires large, shamcontrolled studies and may need to introduce new stimulation parameters. In addition, the combination of rTMS with other traditional therapeutic methods such as medications and deep brain stimulation may lead to new treatment strategies for neurodegenerative diseases.

\section{Abbreviations \\ AD: Alzheimer's disease; ALS: amyotrophic lateral sclerosis; GABA: gamma- aminobutyric acid; HD: Huntington's disease; M1: primary motor cortex; MCl: mild cognitive impairment; MEP: motor evoked potential; PD: Parkinson's disease; rTMS: repetitive transcranial magnetic stimulation; SAl: short latency afferent inhibition; SICl: short interval intracortical inhibition; TMS: transcranial magnetic stimulation.}

\section{Competing interests}

The authors declare that they have no competing interests.

\section{Authors' contributions}

Both authors (Dr. ZN and Dr. RC) drafted the manuscript, read and approved the final version of the manuscript.

Received: 16 September 2015 Accepted: 10 November 2015 Published online: 16 November 2015

\section{References}

1. Lang AE, Lozano AM. Parkinson's disease. First of two parts. N Engl J Med. 1998;339:1044-53.

2. Querfurth HW, LaFerla FM. Alzheimer's disease. N Engl J Med. 2010;362:329-44.

3. Kiernan MC, Vucic S, Cheah BC, Turner MR, Eisen A, Hardiman O, et al. Amyotrophic lateral sclerosis. Lancet. 2011;377:942-55.

4. The Huntington's Disease Collaborative Research Group (MacDonald ME, et al.) A novel gene containing a trinucleotide repeat that is expanded and unstable on Huntington's disease chromosomes. Cell. 1993. 72:971-83.

5. Barker AT, Jalinous R, Freeston IL. Non-invasive stimulation of the human motor cortex. Lancet. 1985;ll:1106-7.

6. Chen R, Cros D, Curra A, Di Lazzaro V, Lefaucheur JP, Magistris MR, et al. The clinical diagnostic utility of transcranial magnetic stimulation: report of an IFCN committee. Clin Neurophysiol. 2008:119:504-32.

7. Hallett M. Transcranial magnetic stimulation: a primer. Neuron. 2007;55:187-99.

8. Ni Z, Charab S, Gunraj C, Nelson AJ, Udupa K, Yeh IJ, et al. Transcranial magnetic stimulation in different current directions activates separate cortical circuits. J Neurophysiol. 2011;105:749-56.

9. Wassermann EM. Risk and safety in repetitive transcranial magnetic stimulation: report and suggested guidelines from the International Workshop on the Safety of Repetitive Transcranial Magnetic Stimulation, June 5-7, 1996. Electroencephalography and Clinical Neurophysiology. 1998;108:1-16.

10. Ridding MC, Rothwell JC. Is there a future for therapeutic use of transcranial magnetic stimulation? Nat Rev Neurosci. 2007;8:559-67.

11. Ni Z, Bahl N, Gunraj C, Mazzella F, Chen R. Increased motor cortical facilitation and decreased inhibition in Parkinson disease. Neurology. 2013;80:1746-53.

12. Ridding MC, Inzelberg R, Rothwell JC. Changes in excitability of motor cortical circuitry in patients with Parkinson's disease. Ann Neurol. 1995;37:181-8.
13. Mackinnon CD, Gilley EA, Weis-McNulty A, Simuni T. Pathways mediating abnormal intracortical inhibition in Parkinson's disease. Ann Neurol. 2005:58:516-24.

14. Ellaway PH, Davey NJ, Maskill DW, Dick JP. The relation between bradykinesia and excitability of the motor cortex assessed using transcranial magnetic stimulation in normal and parkinsonian subjects. Electroencephalogr Clin Neurophysiol. 1995;97:169-78.

15. Chen R, Garg RR, Lozano AM, Lang AE. Effects of internal globus pallidus stimulation on motor cortex excitability. Neurology. 2001;56:716-23.

16. Cunic D, Roshan L, Khan FI, Lozano AM, Lang AE, Chen R. Effects of subthalamic nucleus stimulation on motor cortex excitability in Parkinson's disease. Neurology. 2002;58:1665-72.

17. Cantello R, Gianelli M, Bettucci D, Civardi C, DeAngelis MS, Mutani R. Parkinson's disease rigidity: Magnetic motor evoked potentials in a small hand muscle. Neurology. 1991;91:1449-56.

18. Valls-Sole J, Pascual-Leone A, Brasil-Neto JP, Cammarota A, McShane L, Hallett M. Abnormal facilitation of the response to transcranial magnetic stimulation in patients with Parkinson's disease. Neurology. 1994;44:735-41.

19. Werhahn KJ, Kunesch E, Noachtar S, Benecke R, Classen J. Differential effects on motorcortical inhibition induced by blockade of GABA uptake in humans. J Physiol. 1999:517:591-7.

20. Cantello R. Applications of transcranial magnetic stimulation in movement disorders. J Clin Neurophysiol. 2002:19:272-93.

21. Lee RG, Stein RB. Resetting of tremor by mechanical perturbations: a comparison of essential tremor and parkinsonian tremor. Ann Neurol. 1981;10:523-31.

22. Pascual-Leone A, Valls-Sole J, Toro C, Wassermann EM, Hallett M. Resetting of essential tremor and postural tremor in Parkinson's disease with transcranial magnetic stimulation. Muscle Nerve. 1994;17:800-7.

23. Ni Z, Pinto AD, Lang AE, Chen R. Involvement of the cerebellothalamocortical pathway in Parkinson disease. Ann Neurol. 2010;68:816-24.

24. Kujirai T, Caramia MD, Rothwell JC, Day BL, Thompson PD, Ferbert A, et al. Corticocortical inhibition in human motor cortex. J Physiol. 1993;471:501-19.

25. Ziemann U, Lönnecker S, Steinhoff BJ, Paulus W. The effect of lorazepam on the motor cortical excitability in man. Exp Brain Res. 1996;109:127-35.

26. Ilic TV, Meintzschel F, Cleff U, Ruge D, Kessler KR, Ziemann U. Short-interval paired-pulse inhibition and facilitation of human motor cortex: the dimension of stimulus intensity. J Physiol (Lond). 2002;545:153-67.

27. Müller-Dahlhaus F, Liu Y, Ziemann U. Inhibitory circuits and the nature of their interactions in the human motor cortex a pharmacological TMS study. J Physiol. 2008;586:495-514.

28. Chu J, Wagle-Shukla A, Gunraj C, Lang AE, Chen R. Impaired presynaptic inhibition in the motor cortex in Parkinson disease. Neurology. 2009;72:842-9.

29. Kojovic M, Bologna M, Kassavetis P, Murase N, Palomar FJ, Berardelli A, et al. Functional reorganization of sensorimotor cortex in early Parkinson disease. Neurology. 2012;78:1441-8.

30. Kojovic M, Kassavetis P, Bologna M, Parees I, Rubio-Agusti I, Beraredelli A, et al. Transcranial magnetic stimulation follow-up study in early Parkinson's disease: A decline in compensation with disease progression? Mov Disord. 2015;30:1098-106.

31. Ni Z, Gunraj C, Nelson AJ, Yeh IJ, Castillo G, Hoque T, et al. Two phases of interhemispheric inhibition between motor related cortical areas and the primary motor cortex in human. Cereb Cortex. 2009;19:1654-65.

32. Li JY, Espay AJ, Gunraj CA, Pal PK, Cunic DI, Lang AE, et al. Interhemispheric and ipsilateral connections in Parkinson's disease: Relation to mirror movements. Mov Disord. 2007;22:813-21.

33. Sailer A, Molnar GF, Paradiso G, Gunraj CA, Lang AE, Chen R. Short and long latency afferent inhibition in Parkinson's disease. Brain. 2003;126:1883-94.

34. Sailer A, Cunic DI, Paradiso GO, Gunraj CA, Wagle-Shukla A, Moro E, et al. Subthalamic nucleus stimulation modulates afferent inhibition in Parkinson disease. Neurology. 2007:68:356-63.

35. Wagle-Shukla A, Moro E, Gunraj C, Lozano A, Hodaie M, Lang A, et al. Longterm subthalamic nucleus stimulation improves sensorimotor integration and proprioception. J Neurol Neurosurg Psychiatry. 2013;84:1020-8.

36. Kuriakose R, Saha U, Castillo G, Udupa K, Ni Z, Gunraj C, et al. The nature and time course of cortical activation following subthalamic stimulation in Parkinson's disease. Cereb Cortex. 2010;20:1926-36.

37. Stefan K, Kunesch E, Cohen LG, Benecke R, Classen J. Induction of plasticity in the human motor cortex by paired associative stimulation. Brain. 2000;123:572-84 
38. Morgante F, Espay AJ, Gunraj C, Lang AE, Chen R. Motor cortex plasticity in Parkinson's disease and levodopa-induced dyskinesias. Brain. 2006;129:1059-69.

39. Kim SJ, Udupa K, Ni Z, Moro E, Gunraj C, Mazzella F, et al. Effects of subthalamic nucleus stimulation on motor cortex plasticity in Parkinson disease. Neurology. 2015:85:425-32.

40. Huang YZ, Edwards MJ, Rounis E, Bhatia KP, Rothwell JC. Theta burst stimulation of the human motor cortex. Neuron. 2005;45:201-6.

41. Benninger DH, Berman BD, Houdayer E, Pal N, Luckenbaugh DA, Schneider $L$, et al. Intermittent theta-burst transcranial magnetic stimulation for treatment of Parkinson disease. Neurology. 2011;76:601-9.

42. Zamir O, Gunraj C, Ni Z, Mazzella F, Chen R. Effects of theta burst stimulation on motor cortex excitability in Parkinson's disease. Clin Neurophysiol. 2012;123:815-21.

43. Suppa A, Marsili L, Belvisi D, Conte A, lezzi E, Modugno N, et al. Lack of LTPlike plasticity in primary motor cortex in Parkinson's disease. Exp Neurol. 2011;227:296-301.

44. Elahi B, Elahi B, Chen R. Effect of transcranial magnetic stimulation on Parkinson motor function-systematic review of controlled clinical trials. Mov Disord. 2009;24:357-63.

45. Okabe S, Ugawa Y, Kanazawa I. 0.2-Hz repetitive transcranial magnetic stimulation has no add-on effects as compared to a realistic sham stimulation in Parkinson's disease. Mov Disord. 2003;18:382-8.

46. Zanjani A, Zakzanis KK, Daskalakis ZJ, Chen R. Repetitive transcranial magnetic stimulation of the primary motor cortex in the treatment of motor signs in Parkinson's disease: A quantitative review of the literature. Mov Disord. 2015;30:750-8.

47. Chen R, Classen J, Gerloff C, Celnik P, Wassermann EM, Hallett M, et al. Depression of motor cortex excitability by low-frequency transcranial magnetic stimulation. Neurology. 1997;48:1398-403.

48. Wagle-Shukla A, Angel MJ, Zadikoff C, Enjati M, Gunraj C, Lang AE, et al. Lowfrequency repetitive transcranial magnetic stimulation for treatment of levodopa-induced dyskinesias. Neurology. 2007;68:704-5.

49. Filipovic SR, Rothwell JC, van de Warrenburg BP, Bhatia K. Repetitive transcranial magnetic stimulation for levodopa-induced dyskinesias in Parkinson's disease. Mov Disord. 2009;24:246-53.

50. Hamada M, Ugawa Y, Tsuji S. High-frequency rTMS over the supplementary motor area for treatment of Parkinson's disease. Mov Disord. 2008;11:1524-31.

51. Koch G, Brusa L, Carrillo F, Lo GE, Torriero S, Oliveri M, et al. Cerebellar magnetic stimulation decreases levodopa-induced dyskinesias in Parkinson disease. Neurology. 2009;73:113-9.

52. Mir P, Matsunaga K, Gilio F, Quinn NP, Siebner HR, Rothwell JC. Dopaminergic drugs restore facilitatory premotor-motor interactions in Parkinson disease. Neurology. 2005;64:1906-12.

53. Coyle JT, Price DL, DeLong MR. Alzheimer's disease: a disorder of cortical cholinergic innervation. Science. 1983;219:1184-90.

54. Di Lazzaro V, Oliviero A, Tonali PA, Marra C, Daniele A, Profice P, et al. Noninvasive in vivo assessment of cholinergic cortical circuits in $A D$ using transcranial magnetic stimulation. Neurology. 2002;59:392-7.

55. Pierantozzi M, Panella M, Palmieri MG, Koch G, Giordano A, Marciani MG, et al. Different TMS patterns of intracortical inhibition in early onset Alzheimer dementia and frontotemporal dementia. Clin Neurophysiol. 2004;115:2410-8.

56. Sakuma K, Murakami T, Nakashima K. Short latency afferent inhibition is not impaired in mild cognitive impairment. Clin Neurophysiol. 2007;118:1460-3.

57. Pepin $J$, Bogacz D, De PV, Delwaide P. Motor cortex inhibition is not impaired in patients with Alzheimer's disease: evidence from paired transcranial magnetic stimulation. J Neurol Sci. 1999;170:119-23.

58. Ferreri F, Pauri F, Pasqualetti P, Fini R, Dal Forno G, Rossini PM. Motor cortex excitability in Alzheimer's disease: a transcranial magnetic stimulation study. Ann Neurol. 2003:53:102-8.

59. Perretti A, Grossi D, Fragassi N, Lanzillo B, Nolano M, Pisacreta Al, et al. Evaluation of the motor cortex by magnetic stimulation in patients with Alzheimer disease. J Neurol Sci. 1996;135:31-7.

60. Di Lazzaro V, Pilato F, Dileone M, Saturno E, Profice P, Marra C, et al. Functional evaluation of cerebral cortex in dementia with Lewy bodies. Neuroimage. 2007;37:422-9.

61. Marra C, Quaranta D, Profice P, Pilato F, Capone F, lodice F, et al. Central cholinergic dysfunction measured "in vivo" correlates with different behavioral disorders in Alzheimer's disease and dementia with Lewy body. Brain Stimul. 2012;5:533-8.
62. Nardone R, Bergmann JM,K, Kunz A, Klein S, Caleri F, et al. Abnormal short latency afferent inhibition in early Alzheimer's disease: a transcranial magnetic demonstration. J Neural Transm. 2008;115:1557-62.

63. Nardone R, Bergmann J, Christova M, Caleri F, Tezzon FG,L, et al. Short latency afferent inhibition differs among the subtypes of mild cognitive impairment. J Neural Transm. 2012;119:463-71.

64. Tsutsumi R, Hanajima R, Hamada M, Shirota Y, Matsumoto H, Terao Y, et al. Reduced interhemispheric inhibition in mild cognitive impairment. Exp Brain Res. 2012;218:21-6.

65. Liepert J, Bar KJ, Meske U, Weiller C. Motor cortex disinhibition in Alzheimer's disease. Clin Neurophysiol. 2001;112:1436-41.

66. Inghilleri M, Conte A, Frasca V, Scaldaferri N, Gilio F, Santini M, et al. Altered response to rTMS in patients with Alzheimer's disease. Clin Neurophysiol. 2006;117:103-9.

67. Battaglia F, Wang HY, Ghilardi MF, Gashi E, Quartarone A, Friedman E, et al. Cortical plasticity in Alzheimer's disease in humans and rodents. Biol Psychiatry. 2007;62:1405-12.

68. Koch G, Di Lorenzo F, Bonnì S, Ponzo V, Caltagirone C, Martorana A. Impaired LTP- but not LTD-like cortical plasticity in Alzheimer's disease patients. J Alzheimers Dis. 2012;31:593-9.

69. Koch G, Esposito Z, Codecà CF, Mori F, Kusayanagi H, Monteleone F, et al. Altered dopamine modulation of LTD-like plasticity in Alzheimer's disease patients. Clin Neurophysiol. 2011;122:703-7.

70. Sperling RA, Dickerson BC, Pihlajamaki M, Vannini P, LaViolette PS, Vitolo OV, et al. Functional alterations in memory networks in early Alzheimer's disease. Neruomol Med. 2010;12:22-43.

71. Cotelli M, Manenti R, Cappa SF, Zanetti O, Miniussi C. Transcranial magnetic stimulation improves naming in Alzheimer disease patients at different stages of cognitive decline. Eur J Neurol. 2008;15:1286-92.

72. Cotelli M, Calabria M, Manenti R, Rosini S, Zanetti O, Cappa SF, et al. Improved language performance in Alzheimer disease following brain stimulation. J Neurol Neurosurg Psychiatry. 2011;82:794-7.

73. Ahmed MA, Darwish ES, Khedr EM, El Serogy YM, Ali AM. Effects of low versus high frequencies of repetitive transcranial magnetic stimulation on cognitive function and cortical excitability in Alzheimer's dementia. J Neurol. 2012;259:83-92.

74. Turriziani P, Smirni D, Zappalà G, Mangano GR, Oliveri M, Cipolotti, L. Enhancing memory performance with rTMS in healthy subjects and individuals with Mild Cognitive Impairment: the role of the right dorsolateral prefrontal cortex. Front Hum Neurosci 2012. 6:doi: 10.3389/fnhum.2012.00062.

75. Eisen A, Shytbel W, Murphy K, Hoirch M. Cortical magnetic stimulation in amyotrophic lateral sclerosis. Muscle Nerve. 1990;13:146-51.

76. Mills KR, Nithi KA. Corticomotor threshold is reduced in early sporadic amyotrophic lateral sclerosis. Muscle Nerve. 1997;20:1137-41.

77. Vucic S, Kiernan MC. Novel threshold tracking techniques suggest that cortical hyperexcitability is an early feature of motor neuron disease. Brain. 2006;129:2436-46.

78. Vucic S, Nicholson GA, Kiernan MC. Cortical hyperexcitability may precede the onset of familial amyotrophic lateral sclerosis. Brain. 2008;131:1540-50.

79. Stefan K, Kunesch E, Benecke R, Classen J. Effects of riluzole on cortical excitability in patients with amyotrophic lateral sclerosis. Ann Neurol. 2001;49:536-9.

80. Nihei K, McKee AC, Kowall NW. Patterns of neuronal degeneration in the motor cortex of amyotrophic lateral sclerosis patients. Acta Neuropathol. 1993;86:55-64.

81. Karandreas N, Papadopoulou M, Kokotis P, Papapostolou A, Tsivgoulis G, Zambelis T. Impaired interhemispheric inhibition in amyotrophic lateral sclerosis. Amyotrophic Lateral Sclerosis. 2007;8:112-8.

82. Zanette G, Forgione A, Manganotti P, Fiaschi A, Tamburin S. The effect of repetitive transcranial magnetic stimulation on motor performance, fatigue and quality of life in amyotrophic lateral sclerosis. J Neurol Sci. 2008;270:18-22.

83. Di Lazzaro V, Oliviero A, Saturno E, Pilato F, Dileone M, Sabatelli M, et al. Motor cortex stimulation for amyotrophic lateral sclerosis. Time for a therapeutic trial? Clin Neurophysiol. 2004;115:1479-85.

84. Di Lazzaro V, Dileone M, Pilato F, Profice P, Cioni B, Meglio M, et al. Longterm motor cortex stimulation for amyotrophic lateral sclerosis. Brain Stimul. 2010;3:22-7.

85. Di Lazzaro V, Dileone M, Pilato F, Profice P, Ranieri F, Musumeci G, et al. Repetitive transcranial magnetic stimulation for ALS. A preliminary controlled study. Neurosci Lett. 2006;408:135-40. 
86. Di Lazzaro V, Pilato F, Profice P, Ranieri F, Musumeci G, Florio L, et al. Motor cortex stimulation for ALS: a double blind placebo-controlled study. Neurosci Lett. 2009;464:18-21.

87. Schippling S, Schneider SA, Bhatia KP, Münchau A, Rothwell JC, Tabrizi SJ, et al. Abnormal motor cortex excitability in preclinical and very early Huntington's disease. Biol Psychiatry. 2009;65:959-65.

88. Orth M, Schippling S, Schneider SA, Bhatia KP, Talelli PSJT, et al. Abnormal motor cortex plasticity in premanifest and very early manifest Huntington disease. J Neurol Neurosurg Psychiatry. 2010;81:267-70.

89. Priori A, Polidori L, Rona S, Manfredi M, Berardelli A. Spinal and cortical inhibition in Huntington's chorea. Mov Disord. 2000;15:938-46.

90. Lefaucheur JP, Ménard-Lefaucheur I, Maison P, Baudic S, Cesaro P, Peschanski $M$, et al. Electrophysiological deterioration over time in patients with Huntington's disease. Mov Disord. 2006;21:1350-4

91. Hanajima R, Ugawa Y, Terao Y, Furubayashi T, Machii K, Shiio Y, et al. Intracortical inhibition of the motor cortex is normal in chorea. J Neurol Neurosurg Psychiatry. 1999;66:783-6.

92. Lorenzano C, Dinapoli L, Gilio F, Suppa A, Bagnato S, Currà A, et al. Motor cortical excitability studied with repetitive transcranial magnetic stimulation in patients with Huntington's disease. Clin Neurophysiol. 2006;117:1677-81.

93. Crupi D, Ghilardi MF, Mosiello C, Di Rocco A, Quartarone A, Battaglia F. Cortical and brainstem LTP-like plasticity in Huntington's disease. Brain Res Bull. 2008;75:107-14.

94. Brusa L, Versace V, Koch G, Bernardi G, lani C, Stanzione P, et al. Improvement of choreic movements by $1 \mathrm{~Hz}$ repetitive transcranial magnetic stimulation in Huntington's disease patients. Ann Neurol. 2005;58: 655-6.

95. Di Lazzaro V, Dileone M, Pilato F, Contarino MF, Musumeci G, Bentivoglio $A R$, et al. Repetitive transcranial magnetic stimulation of the motor cortex for hemichorea. J Neurol Neurosurg Psychiatry. 2006;77:1095-7.

96. Obeso JA, Rodriguez-Oroz MC, Goetz CG, Marin C, Kordower JH, Rodriguez $M$, et al. Missing pieces in the Parkinson's disease puzzle. Nat Med. 2010;16:653-61.

\section{Submit your next manuscript to BioMed Central and take full advantage of:}

- Convenient online submission

- Thorough peer review

- No space constraints or color figure charges

- Immediate publication on acceptance

- Inclusion in PubMed, CAS, Scopus and Google Scholar

- Research which is freely available for redistribution 